\title{
A Hybrid High-Resolution Anatomical MRI Atlas with Sub-parcellation of Cortical Gyri using Resting fMRI
}

Anand A. Joshi ${ }^{1}$, Soyoung Choi ${ }^{1,2}$, Minqi Chong ${ }^{1}$, Gaurav Sonkar ${ }^{3}$, Jorge Gonzalez-Martinez ${ }^{4}$, Dileep Nair ${ }^{4}$, Jessica L. Wisnowski ${ }^{6}$, Justin P. Haldar ${ }^{1}$, David W. Shattuck ${ }^{5}$, Hanna Damasio ${ }^{6}$, Richard M. Leahy ${ }^{1}$

${ }^{1}$ Signal and Image Processing Institute, University of Southern California, Los Angeles, USA

${ }^{2}$ Neuroscience Graduate Program, University of Southern California, Los Angeles, USA

${ }^{3}$ Dept. of Computer Science, National Institute of Technology Warangal, India

${ }^{4}$ Epilepsy Center, Cleveland Clinic Foundation, Cleveland, OH, USA

${ }^{5}$ Ahmanson-Lovelace Brain Mapping Center, University of California, Los Angeles, CA, USA

${ }^{6}$ Dornsife Cognitive Neuroscience Imaging Center, University of Southern California, Los Angles, USA 


\begin{abstract}
Brain atlases provide reference parcellations of the brain that are essential for population neuroimaging studies. We present a new high-resolution, single-subject atlas labeled into two parcellation schemes: 1) the anatomical BCI-DNI atlas, which was labeled based on known morphological and anatomical features; and 2) the hybrid USCBrain atlas, which used additional functional information to guide the sub-parcellation of cerebral cortex. Particular attention was paid to the image acquisition, processing and labeling methods to capture fine anatomical details, accommodating for the high-quality data common in recent imaging studies.
\end{abstract}

A single-subject, high-resolution T1-weighted image was acquired and was then processed by an expert neuroanatomist using semi-automated methods in BrainSuite. The brain's features were meticulously extracted with manual corrections to bias-field and masking steps, thereby providing accurate tissue classification and anatomical surface modeling. Guided by sulcal and gyral landmarks, labeled anatomical regions were drawn manually on coronal single-slice images to generate the BCI-DNI atlas, which contains 66 cortical and 29 noncortical regions. The cortical regions were further sub-parcellated based on connectivity analysis of resting fMRI data from multiple subjects in the Human Connectome Project (HCP) database, which were coregistered to the single subject. The resulting USCBrain atlas contains a total of 130 cortical and 29 noncortical regions. In addition to the anatomical and functional parcellations, we also provide a delineation between sulcal valleys and gyral crowns, which offer an additional set of 26 sulcal subregions per hemisphere.

The intended use of the USCBrain atlas is to label individual brains through structural coregistration. To assess utility, we computed the adjusted Rand indices between individual subparcellations obtained through structural-only coregistration to the USCBrain atlas and subparcellations obtained directly from each subject's resting fMRI data. The gyral sub-parcellations generated by atlas-based registration show variable but generally good overlap with the resting fMRI-based subdivisions. In addition to the crisp parcellations, a probabilistic map is included to provide users a quantitative measure of reliability for each gyral subdivision. Both atlases can be used with the BrainSuite, FreeSurfer, and FSL software packages.

Keywords: brain atlas, anatomical MRI, functional MRI, neuroanatomy, segmentation 


\section{INTRODUCTION}

Atlas-based identification of neuroanatomy plays an integral role in studies of neurological disease, cognitive neuroscience, and development and aging, as well as in clinical applications including stereotaxic neurosurgery planning (Dickie et al., 2017). The most commonly used form of atlas registration aligns the anatomical images of single subjects to a reference image based on brain anatomy. Anatomical MRI enables visualization of regions through contrasting image intensities between grey matter, white matter, and cerebral spinal fluid (CSF). Furthermore, it enables identification of the major sulci and associated gyral subdivisions, providing the morphological details required for both anatomical registration and brain segmentation. Once registered, labels from an atlas can be transferred to the MRIs of individual subjects for automated segmentation of the brain into regions of interest (ROIs). Advanced imaging methods have improved the anatomical details that can be captured with high-resolution imaging, enabling more accurate alignment and precise identification of anatomical boundaries.

Multiple approaches have been developed to delineate regions of the brain based on its structural, functional and cellular architecture. Manual labeling of the brain based on anatomical landmarks, e.g., sulci and gyri, remains the most common method of parcellating the brain for atlas creation. Single-subject templates, such as the Tailarach Atlas and the Automated Anatomical Labeling (AAL) Atlas, were parcellated using anatomical images of a single subject (Collins et al., 1995; Talairach and Tournoux, 1988; Tzourio-Mazoyer et al., 2002). Alternative imaging methods have been used to identify cytoarchitectonic, myeloarchitectonic, or functional features, which are used in turn to parcellate the brain (Chakravarty et al., 2006; Chong et al., 2017; Essen and Drury, 1997; Yeo et al., 2011; Zilles and Amunts, 2010). Diffusion MRI has been used to parcellate the cortex based on differences in myelination within distinct brain regions, as well as through delineation of regions based on structural connectivity between these regions (Glasser et al., 2014). Similarly, functional MRI (fMRI) can be used to identify contiguous regions of cortex that show similar functional properties (Bhushan et al., 2016; Joshi et al., 2018; Margulies et al., 2009). Parcellation methods based on fMRI include region growing (Blumensath et al., 2012), graph cut (Chong et al., 2017; Li et al., 2018; Shi and Malik, 2005), hierarchical clustering (Cordes et al., 2002) and ICA (Calhoun et al., 2009). Individual variations and limited data from single-subject templates may not be representative of the larger population. To address individual variation, multi-subject templates, including average brain and probabilistic atlases, attempt to account for the variability of brain architecture across a population (Desikan et al., 2006; Fischl, 2012; Mazziotta et al., 1995). Multi-subject functional atlases are created by anatomical coregistration onto a common space followed by group parcellation based on fMRI (Chong et al., 2017; Craddock et al., 2013; Glasser et al., 2016; Yeo et al., 2011). Lastly, multi-modal combined with multi-subject approaches have pooled data across subjects and used multiple imaging contrasts to build an atlas with an integrated pattern classification strategy (Glasser et al., 2016). In that work, Glasser and colleagues used a large number of multimodal features from Human Connectome Project (HCP) data to parcellate the cerebral cortex into 180 unique regions per hemisphere (Glasser et al., 2016).

It is important to note that the studies and atlases cited above have not agreed on a common parcellation scheme and each method has its own assumptions. Given the broad array of available techniques and atlases, selection of the most appropriate method of parcellation or choice of atlas depends heavily on the application. Arguments, however, can be made for the continued use of 
anatomical segmentations. For example, a practical challenge in using parcellations that are not based on standard anatomical landmarks is how to compare findings with earlier studies that are based on classically defined gyral labels. Regions in newly defined parcellations can overlap with multiple historically defined regions leading to ambiguity in referencing these regions for metaanalysis. Additionally, anatomically defined labels can be used as a guide for clinicians to identify and delineate regions for surgical planning. For example, sulcal landmarks are the first salient anchors for cortical subdivisions in defining resection targets in neocortical epilepsy. However, limiting the volume of brain tissue surgically resected could help retain more function, but there is insufficient information in structural MRI alone to justify subparcellation beyond delineation of the major gyri. Linking gyral sub-parcellations to functional specialization based on fMRI connectivity could help explain why differing seizure semiologies arise from the same gyrus in patients with epilepsy (Mailo and Tang-Wai, 2015). This in turn could provide surgeons with information to better delineate focal resections by defining boundaries that are more precise in comparison to the standard gyral divisions. Therefore, a parcellation scheme that retains the salient guidance of sulcal landmarks while also using functionally-defined subgyral parcels could provide a principled approach to gyral subdivision while retaining the classically defined gyral boundaries.

In this paper, we present an anatomical atlas (BCI-DNI atlas) and a hybrid anatomical-functional atlas (USCBrain atlas), which are intended to be used for coregistration and labeling of MR images. The BCI-DNI atlas was prepared using a high-resolution 3D T1-weighted (T1W) MRI images of a single subject. The atlas was meticulously extracted and manually delineated with anatomical labels based on known sulcal and gyral landmarks (Damasio, 2005; Pantazis et al., 2010). It was designed to be used with a robust registration algorithm that attempts to use maximal anatomical information for alignment of both cortical surface and volumetric anatomy, such as those provided by BrainSuite or FreeSurfer (Joshi et al., 2012b, 2007; Postelnicu et al., 2009). MR imaging techniques continue to improve, allowing the brain's anatomy to be captured at increasingly higher resolution. Use of a high-resolution atlas may help improve accuracy and reproducibility of registration and segmentation as the techniques available for researchers and clinicians continue to grow along this trajectory.

Additionally, we have developed a principled approach to sub-parcellating anatomically-defined ROIs based on rfMRI data. The intended goal of the resulting USCBrain atlas is to define functionally distinct subdivisions of the gyri defined in the BCI-DNI atlas. Use of functional data enables gyral subdivision in the absence of anatomical landmarks. We used resting fMRI (rfMRI) signals to compute connectivity activity between neighbouring vertices to identify boundaries between functionally distinct regions within each gryal ROI. At very fine scales, we expect the boundaries of these functional regions to vary significantly with respect to individual sulcal anatomy (Miller et al., 2009; Ono et al., 1990). We therefore limit the number of subdivisions of each gyrus $(\leq 4)$ based on a Silhouette score (Rousseeuw, 1987) and use an independent data set from 60 subjects to evaluate the consistency of the resulting subdivisions across subjects. Each label was named by combining its anatomically defined ROI and its location within the ROI (e.g., anterior-, middle-, or posterior- cingulate gyrus). The rfMRI analysis and parcellation were performed on the cortical surface. The cortical surface parcellation was then mapped back to the original volume, resulting in an atlas with mutually consistent volumetric and cortical surface labels. A probabilistic atlas was also generated for the USCBrain atlas. In addition to the anatomical and functional parcellations, we also provide a delineation between sulcal valleys and 
gyral crowns which offer an additional set of 26 sulcal subregions per hemisphere. Both parcellations in the atlas have general applicability to cognitive neuroscience and clinical research studies. The BCI-DNI and USCBrain atlases are available for download and use with our opensource software, BrainSuite as well as with FreeSurfer and FSL, to coregister and label MR images (http://brainsuite.org/atlases).

\section{MATERIALS AND METHOD}

\subsection{The BCI-DNI Anatomical Brain Atlas}

\subsubsection{Image Acquisition}

We acquired a high-resolution 3D MPRAGE image (TE=4.33 ms; TR=2070 ms; TI=1100ms; Flip angle $=12$ degrees; resolution $=0.547 \times 0.547 \times 0.802 \mathrm{~mm}$ ) on a 3 T Siemens MAGNETOM Trio using a 32 channel head coil. Fat suppression was achieved using spectrally-selective excitation of the water protons. Data was acquired 5 times and averaged to improve SNR at this resolution. The subject was a typical right-handed woman in her mid-thirties. Her brain is brachycephalic with no obvious anomalies.

\subsubsection{Brain Extraction}

T1-weighted images were processed using BrainSuite (http://brainsuite.org/) in a semi-automated fashion to classify tissue types, extract and render the surfaces of the inner, mid and pial cortices (Shattuck and Leahy, 2002). Bias field correction was performed using both automatic processing and manual guidance to maximize grey-white tissue contrast and ensure accurate tissue classification (Shattuck et al., 2001). Manual edits were performed on the cortical boundaries to correct inclusion of meninges or exclusion of cortex, on the occipito-cerebellar boundaries to correct inclusion of cerebellum or tentorium or exclusion of the occipital tissue, and on the greywhite boundaries to preserve fine sulcal and gyral detail.

\subsubsection{Anatomical Labeling}

Anatomical labeling was performed manually on coronal single-slice images. Sixty-six cortical regions of interest (ROIs) were delineated manually using sulcal and gyral landmarks for guidance as detailed in (Damasio, 2005). Volumetrically, deep gyral boundaries were defined on the atlas based on the two opposing sulcal edges of the gyrus from the coronal view. Cortical volume labels were then interpolated and transferred to the mid-cortical surface meshes for the left and right hemispheres. Cortical surface labels were refined so that label boundaries would follow the sulcal fundi more closely using the geodesic curvature flow method described in (Joshi et al., 2012a), and were then transferred back to the volume label set for consistency between surface mesh labels 
and volumetric labels. A final manual editing of the labels was performed on both the cortical surface and volumetric labels. Subcortical nuclei, hippocampus, amygdala, corpus callosum, ventricles, brainstem, cerebellum, and other noncortical regions were all labeled manually with no automated refinements. Figure 1 shows the colorcoded BCI-DNI atlas with labels, and Table 1 in the Appendix lists the full set of ROIs labeled in the atlas.

Twenty six sulcal curves were delineated on the midcortical surfaces using our sulcal tracing protocol

(http://neuroimage.usc.edu/CurveProtocol.html) (Joshi et al., 2010). Additional sulci were marked on the second set of curves, totaling 39 sulci on the left hemisphere and 37 sulci on the right hemisphere (Table 2 in the Appendix). The 13 (right) and 11 (left) additional sulci are not included in our sulcal tracing protocol but are still commonly found. (Damasio, 2005; Duvernoy, 1999; Pantazis et al., 2010)

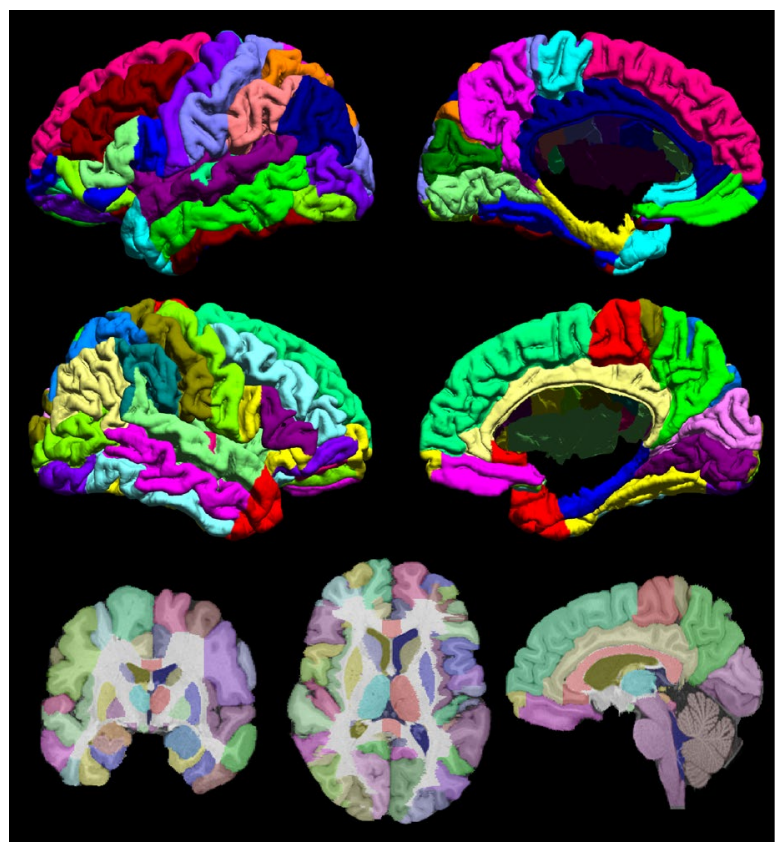

Figure 1. The BCI-DNI anatomical atlas with 95 regions of interest (66 cortical, 29 noncortical) manually labeled by an expert neuroanatomist. Labeled left (top row) and right hemisphere (middle row) of the lateral (left column) and mesial (right column) midcortical surfaces. (bottom row) Single-slice skull-stripped MPRAGE image with labels overlaid on coronal (left), axial (middle) and sagittal (right) orientation. 
We also delineated sulcal regions around the 26 sulcal curves for applications where users want to differentiate between sulcal valleys and gyral crowns. The delineations of the sulcal regions were performed for each of the atlas's cortical hemispheres on the mid-cortical surface meshes. The mid-cortical surface representations were smoothed and then mean curvature was computed. For this purpose, first, principal curvatures were computed by fitting quadratic polynomials in each of the vertex neighborhoods (Desbrun and Polthier, 2007). The mean of the two principal curvatures
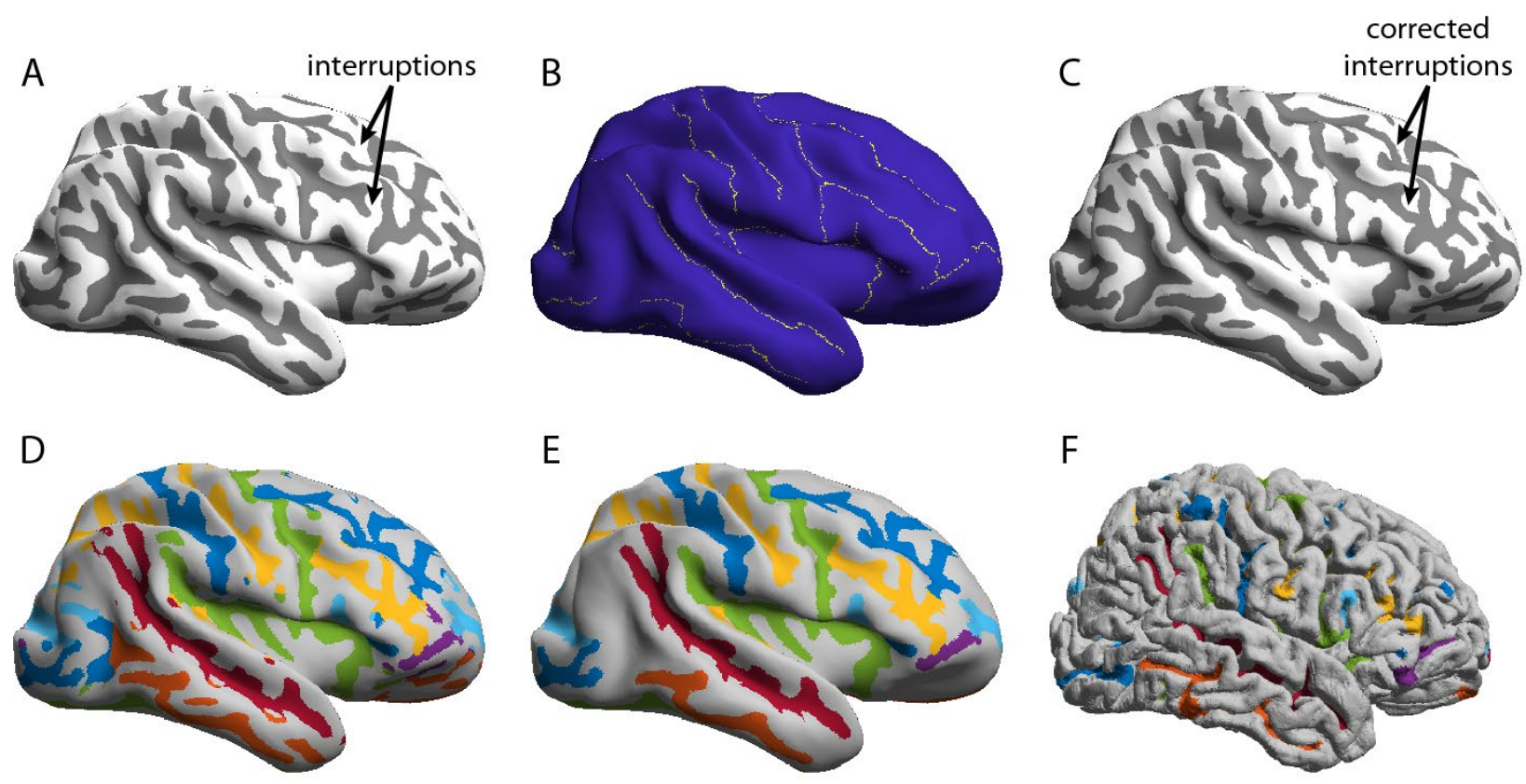

Figure 2. The procedure for delineation of sulcal regions. (a) First, the mean curvature maps were computed and thresholded. (b) The 26 sulcal curves were transferred to the surface. (c) These were used to correct for the interruptions along the sulcus in the curvature maps. (d) The 26 sulcal curves were used to label the individual sulcal regions. (e) These regions were further refined using connected component analysis and morphological smoothing. (f) The same sulcal regions as in (e) shown on the original mid-cortical surface.

results in the mean curvatures (Do Carmo, 2016). Sulcal regions were defined as those with negative mean curvature found by thresholding on the zero level set. The 26 sulcal traces were then transferred to the surfaces. Any interruptions in the sulcal regions along each of the sulci were corrected using nearest neighbor labeling relative to the sulcal curve. Finally, the 26 sulcal regions were identified using connected component analysis for each cortical hemisphere. The process is illustrated in Figure 2.

\subsection{The USCBrain Hybrid Brain Atlas}

The anatomical parcellation defined by the BCI-DNI atlas was subdivided using rfMRI data from the Human Connectome Project (HCP). The BCI-DNI atlas labels were transferred to the HCP grayordinate space (Glasser et al., 2013) in which the HCP's minimally preprocessed rfMRI data were available. After sub-parcellation the labels were transferred back to the original BCI-DNI atlas space. 


\subsubsection{Study Population and Data Preparation}

A 40 subject dataset (healthy adults, ages 22-35, 16 males, 24 females) was obtained from the WU-Minn Human Connectome Project (HCP) database (Glasser et al., 2013; Van Essen et al., 2013). T1W images and four sessions of rfMRI data were utilized for each subject (TR=720ms, $\mathrm{TE}=33.1 \mathrm{~ms}, 2 \times 2 \times 2 \mathrm{~mm}$ voxels, scan time= $15 \mathrm{mins})$. All data was processed using the HCP's minimal pre-processing pipeline (MPP) (Glasser et al., 2013). The pre-processing pipeline includes processing of T1W images of each subject using FreeSurfer (Fischl, 2012) for the identification of cortical surfaces and coregistration of the surfaces to a common atlas. These surfaces were then down-sampled to a common standard cortical surface mesh (32K Conte-69). Resting fMRI data were corrected for acquisition artifacts and subject to a non-aggressive spatiotemporal filtering as described by (Glasser et al., 2013). The time-series data were then linearly resampled onto the mid-cortical surfaces generated by FreeSurfer and transferred to the 32K Conte-69 grayordinate representation (Glasser et al., 2013). We additionally denoised the rfMRI data by applying nonlinear temporal nonlocal-means (tNLM) filtering (Bhushan et al., 2016). The tNLM filtering reduces local signal fluctuations in the fMRI data without the spatial blurring that occurs with standard linear filtering. The resulting time series at each vertex were normalized to zero mean and unit length.

The BCI-DNI atlas was processed through the FreeSurfer pipeline to generate cortical meshes for inner and pial surfaces and to coregister these meshes to the fsaverage brain atlas in FreeSurfer using spherical mapping and curvature-based registration. The BCI-DNI labels from the inner BrainSuite cortical surface were transferred to the inner FreeSurfer cortical surface mesh. This transfer is possible because both software packages generate very similar cortical surfaces. For both cortical hemispheres, using the coregistered spherical maps provided with the HCP data, the labels from the BCI-DNI atlas were transferred to the $32 \mathrm{~K}$ Conte- 69 surface meshes by nearest neighbor interpolation. These spherical maps are used as intermediate representation for cortical surface representation by FreeSurfer as part of the HCP pipeline (Fischl et al., 1999). As a result, for each subject a BCI-DNI-atlas labeled 32K Conte-69 mesh in the grayordinate coordinates was obtained, the same space in which the HCP rfMRI data was also available.

\subsubsection{Resting fMRI Based Sub-Parcellation}

For each ROI from the BCI-DNI atlas in the grayordinate representation (Figures 3a\&b), we computed a similarity matrix for each subject using the rfMRI data. The similarity measure was calculated between each pair of vertices in the ROI using their respective time series $X$ and $Y$ as $s(X, Y)=\pi-\cos ^{-1}(X \cdot Y)$, where the dot-product $X \cdot Y$ indicates the Pearson correlation coefficient between the time-series and $\cos ^{-1}(\cdot)$ represents the principal value of the inverse cosine function. We chose this measure because $\cos ^{-1}(X \cdot Y)$ in the range $[0, \pi)$ represents the geodesic distance between the two unit-length vectors $X$ and $Y$ and is therefore a true metric on the hypersphere. This pairwise similarity matrix was input to the spectral-clustering normalized graph-cut algorithm (Shi and Malik, 2005, Craddock et al., 2013) to subdivide each ROI. The spectral clustering algorithm chooses a set of graph cuts defining the region boundaries that minimizes the ratio of the total edge weight along the cuts to the total edge weight within regions. As a result, the gyrus is subdivided such that the subdivisions have distinct connectivity profiles. 


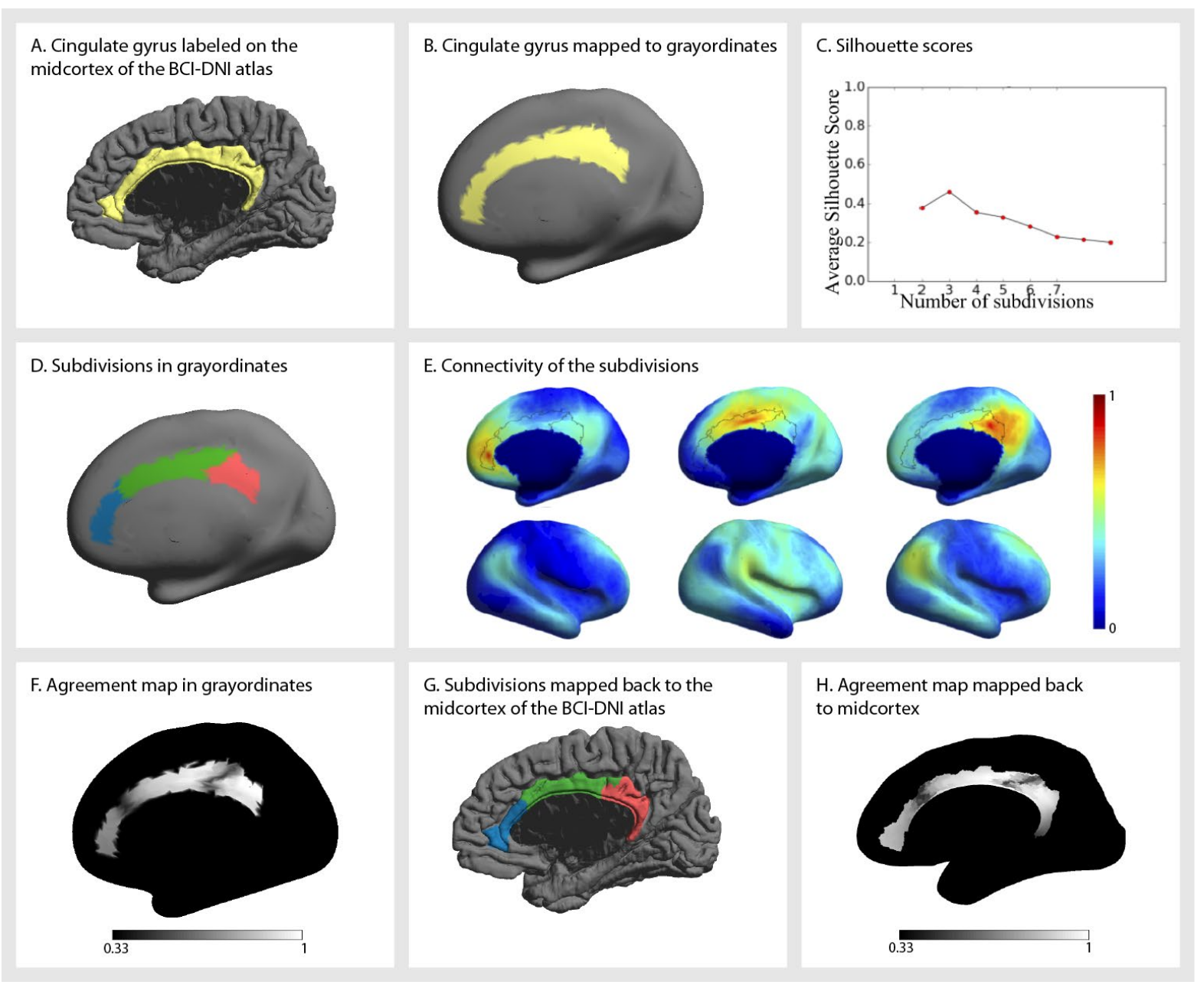

Figure 3. The process of subdivision is shown in the figure with right cingulate as an illustrative example: (a) right cingulate gyrus from BCI-DNI atlas; (b) label transferred to the grayordinate space of HCP in which the fMRI data is available; (c) silhouette score computed for different numbers of clusters; (d) subdivisions performed for one of the 40 subjects; (e) seed-based connectivity using centroid vertex of each subdivision is computed to show differences in connectivity of each subdivision; (f) agreement maps across 40 subjects; ( $g$ ) the subdivision labels transferred back to the midcortex of the BCI-DNI brain; (h) subdivision agreement maps transferred back to the midcortex of the BCI-DNI brain

To determine the optimal number of subdivisions, we computed the silhouette score (Rousseeuw, 1987). This measure compares the similarity of each vertex to other vertices in its own cluster in comparison to all other clusters, and has been previously proposed as an optimality metric that can be used to automatically choose the number of different clusters in general clustering applications (de Amorim and Hennig, 2015). Silhouette scores vary from -1 to +1 , where a large positive value indicates that the vertex matches the assigned cluster well and a small or negative value indicates a poor fit. The average silhouette score over all subjects for a different number of subdivisions $\mathrm{N}$ was computed and the $\mathrm{N}$ that maximized the average silhouette score was chosen for each ROI. For example, Figure $3 \mathrm{c}$ indicates an optimal value of $\mathrm{N}=3$ for right cingulate. 
The sub-parcellations were computed for the $40 \mathrm{HCP}$ subjects. The cluster labels assigned to gyral subdivisions by the clustering algorithm have an arbitrary order and are not consistent across subjects. We used the Hungarian algorithm (Kuhn, 1955) to reorder the cluster labels so that they were maximally consistent across subjects. The final subparcellation of the ROIs was generated by taking a majority vote (random assignment in case of ties) of labels at each vertex over the 40 subjects (Figure 3d). Qualitative verification of the subparcellation was performed by visualizing the functional connectivity patterns between subparcellations of each anatomical ROI. The geometric centroid vertex within a cluster was used as the seed point to compute the correlation of its time series to that of all other vertices throughout the brain. Correlations were averaged across subjects and displayed as illustrated in Figure 3e. To check the consistency of labels across the 40 subjects, we computed a label agreement map as follows. At each vertex, we performed pairwise comparisons of labels between each pair of subjects and assigned a value of 1 if they are same or 0 if they are different. The agreement map was then computed as the average over all possible pairs, consisting of values in the range $(1 / N, 1)$ where $N$ is the number of subdivisions. High agreement scores were expected towards the center of the labels while low scores were expected near the edges. Sharp boundaries between subdivisions are indicative of consistency of subdivisions across subjects and were deemed preferable. A grayscale modulated agreement map is shown for right cingulate in Figure 3(f). We then mapped the labels and agreement maps for each of the subdivided gyri from the $32 \mathrm{~K}$ Conte- 69 inner surface mesh back to the BCI-DNI atlas inner cortical surfaces using nearest neighbor interpolation as described in section 2.2.1 (Figure $3(\mathrm{~g}, \mathrm{~h})$ ). The inner, mid cortical, and pial surfaces generated by BrainSuite have the same number of vertices and are in correspondence with each other. Therefore, labels were copied directly from the inner cortical surface to the mid and pial surfaces.

We note that the optimal number of regions was identical for homologous ROIs in the left and right hemispheres in most cases. For example, for cingulate gyrus (Figure 3) the silhouette scores had a maximum for 3 subdivisions for both hemispheres. However, in some ROIs the optimal number of left and right subdivisions differed, specifically in the angular, middle occipital, middle temporal, and superior temporal gyri. The angular gyrus (Figure 4) showed maximum silhouette scores for 2 (left) and 3 (right) subdivisions respectively. However, on inspection the connectivity maps were distinct, and the agreement maps also showed distinct boundaries for 3 subdivisions in both hemispheres. Therefore, the angular gyrus was subdivided into 3 regions for both hemispheres, as illustrated in Figure 4. A similar process of inspection of the connectivity and agreement maps was used to select equal numbers of subdivisions in left and right homologous regions for the other gyri listed above. In addition, while the inferior temporal gyri had optimal silhouette scores for 2 subdivisions bilaterally, 3 subdivisions were chosen because of the large size of the gyrus and the fact that connectivity and agreement maps indicated a clear and distinct delineation of three regions across subjects. 
As a final step, the refined cortical surface labels in the BCI-DNI atlas space were propagated from the surface onto the volumetric atlas. Two intermediate surfaces (one each between inner/mid and $\mathrm{mid} /$ pial) were generated and labeled, yielding a total of 5 labeled surfaces in the cortical ribbon. The gyral volume labels (grey matter, along with the white matter regions) as defined in the BCIDNI atlas, were filled using nearest neighbor interpolation with these surface labels as a reference. The agreement map was propagated to the volume in a similar manner to retain a one-to-one correspondence between the surface and volume labels as shown in Figure 5. Agreement maps
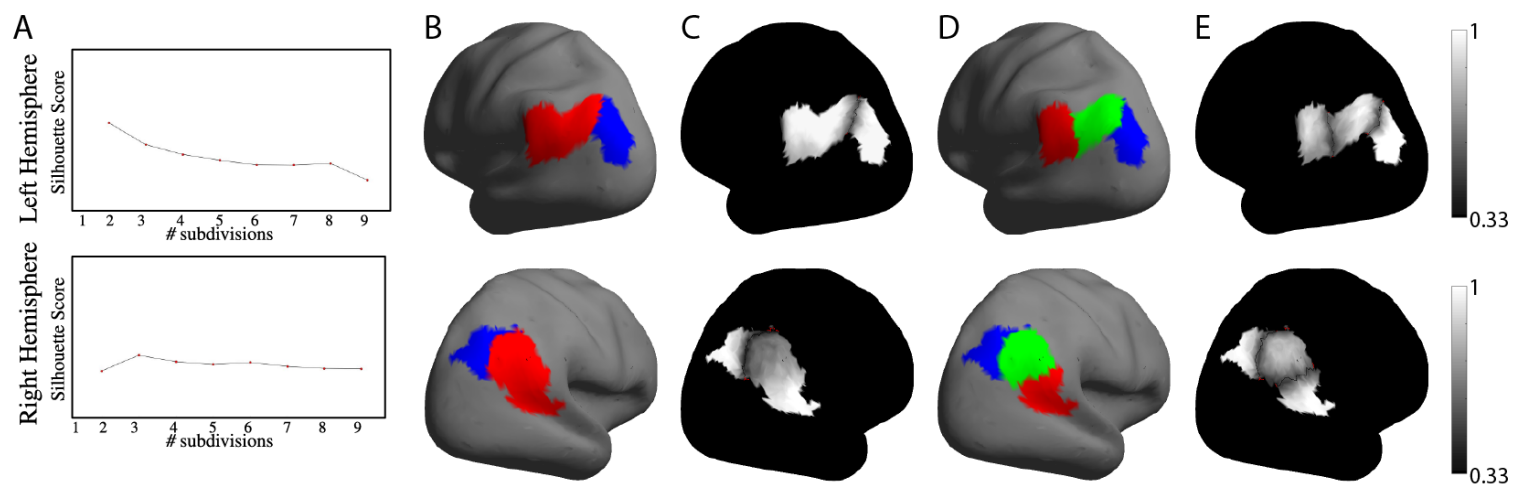

Figure 4: Subdivision of the angular gyrus for left and right hemispheres: (a) silhouette analysis; (b) 2 subdivision labels; (c) the corresponding agreement map; (d) 3 subdivision labels for the same gyrus; and (e) corresponding agreement map. As can be seen, 3 subdivisions resulted in crisper agreement maps for the right hemisphere. For the left hemisphere, while 2 subdivisions showed better agreement maps, 3 subdivisions showed a still acceptable agreement map and a high silhouette score. We therefore choose 3 subdivisions to maintain symmetry between the two hemispheres.

were combined across all cortical gyri to generate probability maps that represent inter-subject agreement of the labels across the 40 subjects at each vertex in the cortex, as shown in Figure 6.

\subsection{Validation}

To evaluate the consistency of labeling of gyral subdivisions using the USCBrain atlas, we investigated how precisely the boundaries of the sub-parcellations could be identified by coregistration of the atlas to subject using T1W MRI data only. The rationale for using the USCBrain atlas-based parcellation instead of individual rfMRI based parcellation is that in most neuroimaging studies, T1W images are acquired with enough anatomical detail for robust registration. Conversely, resting fMRI data are not routinely collected, and even when available they are rarely of the quality for reliable functional segmentation, in terms of resolution, temporalduration, and artifact contamination, as the HCP data used here. Therefore, we investigated whether the cortex can be sub-parcellated into functionally meaningful regions using anatomically driven coregistration alone.

An additional separate set of $60 \mathrm{HCP}$ subjects, with T1W images and four 15-minute rfMRI sessions were selected and processed as described above. We compared consistency between: (a) sub-parcellations by atlas coregistration to the individual T1W image, as described in section 2.2.1; 
and (b) sub-parcellations for that same individual obtained using each of the four sessions of rfMRI data for each subject as described section 2.2.2. We then compared the sub-parcellation results from (a) to the results from (b) using the Adjusted Rand Index (ARI) (Rand, 1971) for each of the 4 sessions and all subjects $(60 * 4=240)$ for all ROIs. The ARI ranges from 0 to 1 , where 0 indicates labeling performance equivalent to random assignment while 1 indicates perfect agreement. We do expect to see intra-subject variability in the functional parcellation for different rfMRI sessions because repeated rfMRI scans within the same subject can capture different connectivity patterns (Miller et al., 2009). The measures of uncertainty in parcellations determined from individual rfMRI data then provides a baseline score with which to compare parcellation based on registration of the USCBrain atlas. If the ARI between atlas coregistration and rfMRI-based sub-parcellations is comparable to the ARI between different sessions of rfMRI, then registration of the atlas would serve as an appropriate surrogate for individual rfMRI based parcellation.

\subsection{Interoperability with BrainSuite, FreeSurfer, and FSL}

The atlases were created to be used with the BrainSuite (http://brainsuite.org), FreeSurfer (https://surfer.nmr.mgh.harvard.edu) and FSL (https://fsl.fmrib.ox.ac.uk/fsl/fslwiki) software packages. BrainSuite and FreeSurfer both use a subject's T1w images as input and generate cortical surface representations. These surfaces are mapped to a flat (for BrainSuite) or spherical (for FreeSurfer) space, and then coregistered in that space to similarly mapped atlas surfaces (Fischl, 2012; Joshi et al., 2012b). FSL, on the other hand, does not generate cortical surface representations, but performs whole brain volumetric registration using 3D nonlinear registration (Andersson et al., 2007). BrainSuite also performs volume registration using a cortically constrained approach so that the cortical surface and volume alignment results are mutually consistent.

The approximate processing time required for registration and labeling on typical image volume (image size: $128 \times 256 \times 256$; resolution: $1.33 \times 1 \times 1 \mathrm{~mm}^{3}$ ) using a desktop workstation (speed Intel Xeon model E5630, 24GB RAM) were, for each software package: 1.5 hours for BrainSuite to perform surface and volume registration and labeling; 20 hours for FreeSurfer to perform surface labeling only; and 1.2 hours for FSL (FLIRT+FNIRT) to perform volume labeling. Scripts and instructions for using the atlases with each software package are available at http://brainsuite.org/using-atlases.

\section{RESULTS}

\subsection{Parcellation}

The BCI-DNI anatomical atlas is shown in Figure 1 with the cortical labels on the mid-cortical surface and the volume labels on the sagittal, coronal and axial slices. A full description of the atlas and downloadable files can be found on our BrainSuite website (http://brainsuite.org/svreg_atlas_description/). This atlas has 95 ROIs delineated in the volume, of which 66 of these regions are cortical subdivisions (33 per hemisphere). The 76 sulcal curves are also included with the atlas. 
The USCBrain atlas is displayed in Figure 5. Of the 33 initial anatomical cortical regions per hemisphere in the original BCI-DNI atlas (Table 1 in the Appendix), 23 ROIs were subdivided, resulting in a total of 65 regions per hemisphere. The remaining 8 original ROIs did not exhibit sufficient internal variation in functional connectivity in their rfMRI data to justify subdivision. Most anatomical ROIs were subparcellated into 2 or 3 regions. As expected, large anatomical ROIs, such as the superior, middle and inferior temporal gyri, middle frontal gyrus, and cingulate cortex, were subdivided into two or more subdivisions, while smaller ROIs, such as the temporal pole, paracentral lobule, and Heschl's gyrus, were not subdivided.

\subsection{Intersubject Label Consistency}

A grayscale modulated image showing agreement in labels across the 40 subjects of the subparcellated atlas generated by majority vote is shown in Figure 6. The value at each voxel reflects the percentage of subjects that matched the label at that voxel. The grayscale ranges from black, indicating no consistency, to white, indicating perfect consistency across subjects. These maps show near-perfect consistencies $(\sim 1)$ near the centers of the sub-parcels, as well as at the

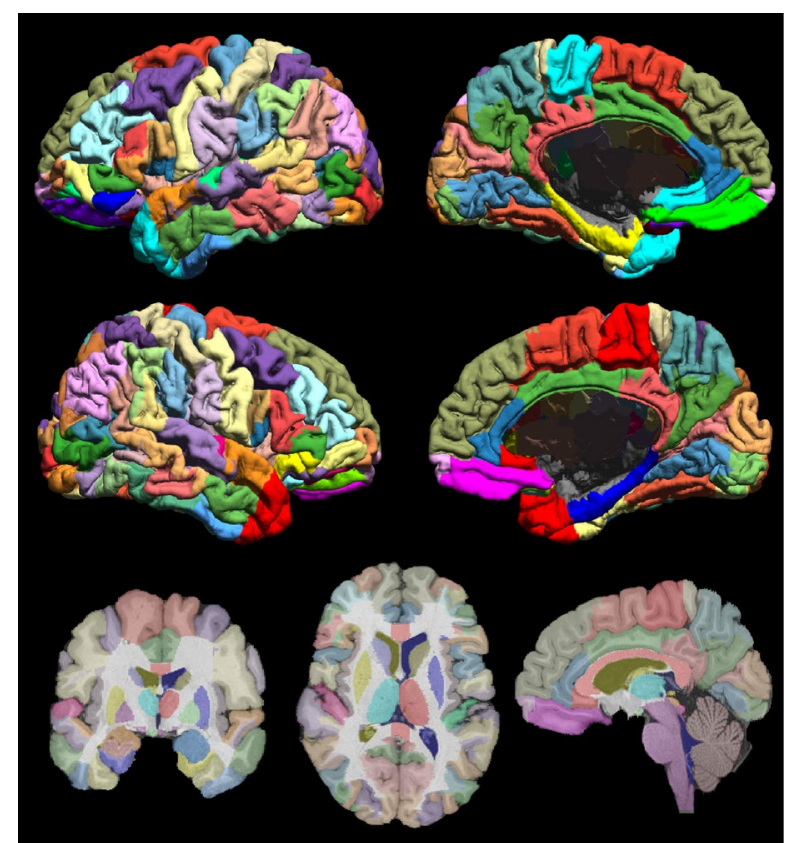

Figure 5. The USCBrain atlas with 130 cortical regions of interests subdivided using rfMRI data. The figure shows the mid-cortical surface of the atlas, color coded for each subparcellated region. Labeled left (top row) and right (bottom row) hemisphere and mesial (right column) and lateral (left column) midcortical surfaces. (bottom row) Single-slice skull-stripped MPRAGE image with labels overlaid on coronal (left), axial (middle) and sagittal (right) orientation. boundaries of anatomical parcels and reduced consistencies $(\sim .5)$ at the boundaries of the functional sub-parcels. A few regions showed higher variability, including the right inferior temporal gyrus and the right angular gyrus, as well as the bilateral inferior occipital gyri, where values were as low as 0.7 in the center of each of these regions, indicating substantial functional variability with respect to the sulcal and gyral anatomy that guides coregistration to the original BCI-DNI atlas. 
bioRxiv preprint doi: https://doi.org/10.1101/2020.09.12.294322; this version posted September 13, 2020. The copyright holder for this preprint (which was not certified by peer review) is the author/funder, who has granted bioRxiv a license to display the preprint in perpetuity. It is made available under aCC-BY-NC-ND 4.0 International license.
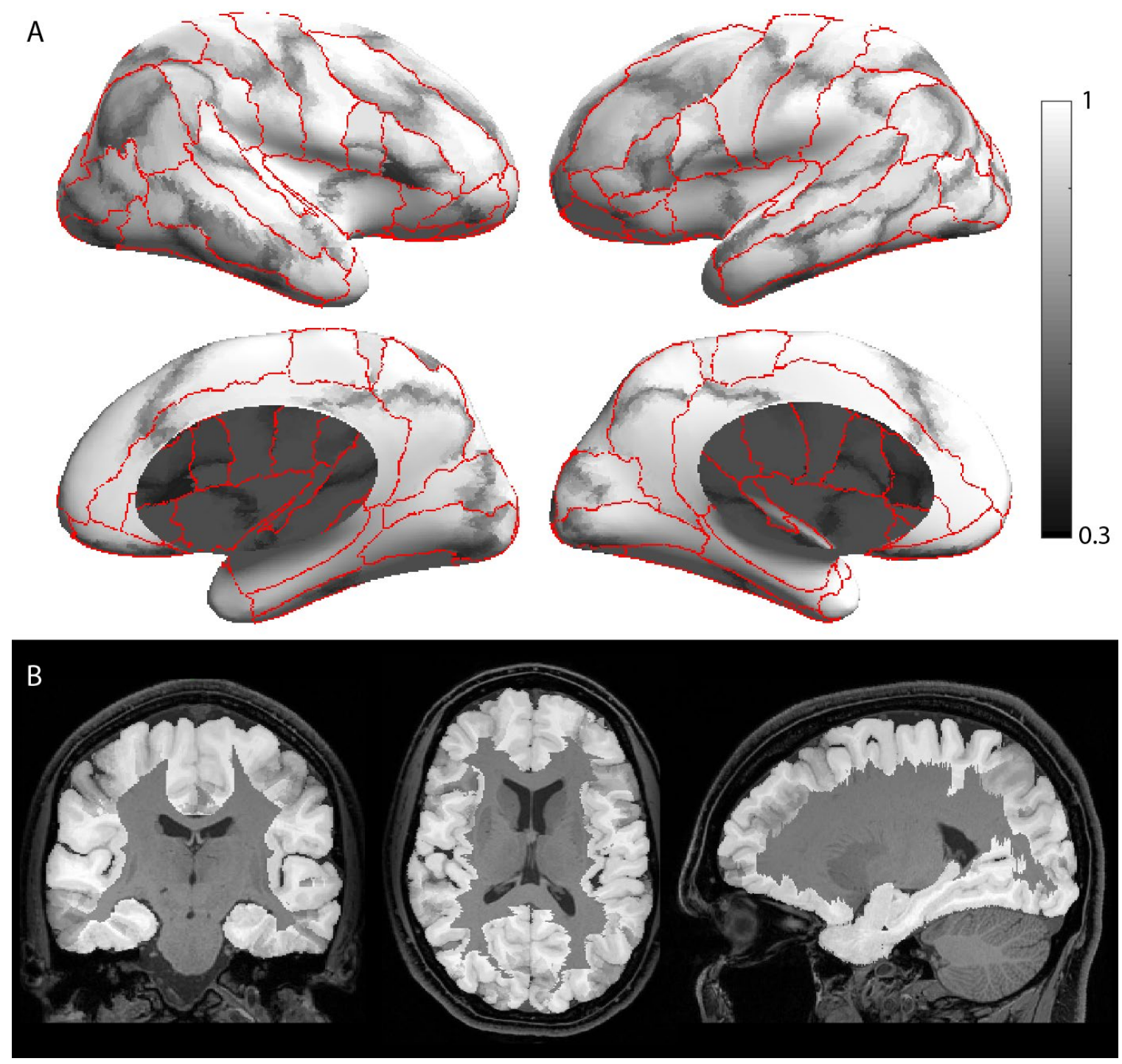

Figure 6. Grayscale modulated plot showing agreement of the USCBrain atlas labels across the 40 subjects. White (value of 1) indicates perfect agreement at that vertex. The probability maps were generated on the surface as shown in (a) and transferred to the volume as shown in (b). Red curves indicate ROI boundaries of the BCI-DNI atlas. 


\subsection{Anatomical vs Functional Labeling}

As noted earlier, we chose an additional set of $60 \mathrm{HCP}$ subjects for the purpose of validation, which were disjoint from the set of 40 subjects used to generate the atlas. Box plots of the ARIs for each of the cortical ROIs in the BCI-DNI atlas are shown in Figure 7. For each ROI, we show consistency between atlas and rfMRI based subparcellation on the left and consistency between different rfMRI sessions on the right. The ROIs that were not subdivided show perfect agreement with an ARI of 1 but are included in the figure for completeness. For the 60 validation subjects, overall the sub-parcellations generated by atlas-based registration relative to rfMRI results showed consistently lower average ARI than those based on comparison of individual rfMRIs from different sessions. With the high quality and 15-minute duration of the HCP data, not surprisingly, using an individual subject's rfMRI data typically generated a better functional parcellation of that subject than using the atlas. However, as noted above, most studies do not routinely acquire comparably high quality rfMRI data. In this case, the atlas-based subparcellations serve as a surrogate for individual rfMRI-based parcellation. The results in Figure 7 show that the majority of regions in the atlas have mean ARIs for atlas vs rfMRI within the inter-quartile range of the rfMRI vs rfMRI results. In some areas consistency is rather poor, including left and right middle orbitofrontal and lingual gyrus, left superior temporal gyrus, left post-central gyrus, angular gyrus, and superior occipital gyrus. These results are discussed in more detail below. 
bioRxiv preprint doi: https://doi.org/10.1101/2020.09.12.294322; this version posted September 13, 2020. The copyright holder for this preprint (which was not certified by peer review) is the author/funder, who has granted bioRxiv a license to display the preprint in perpetuity. It is made available under aCC-BY-NC-ND 4.0 International license.

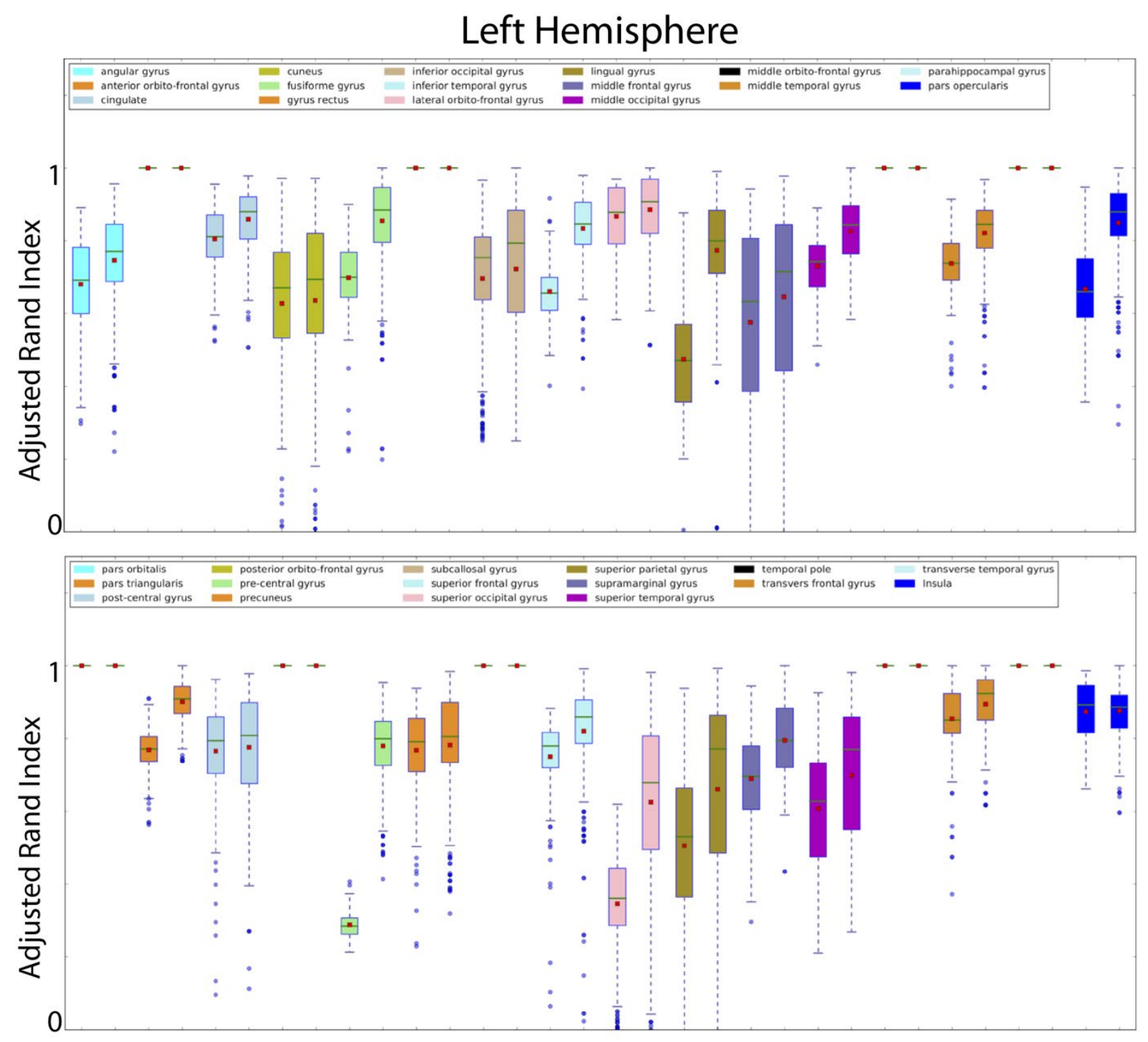



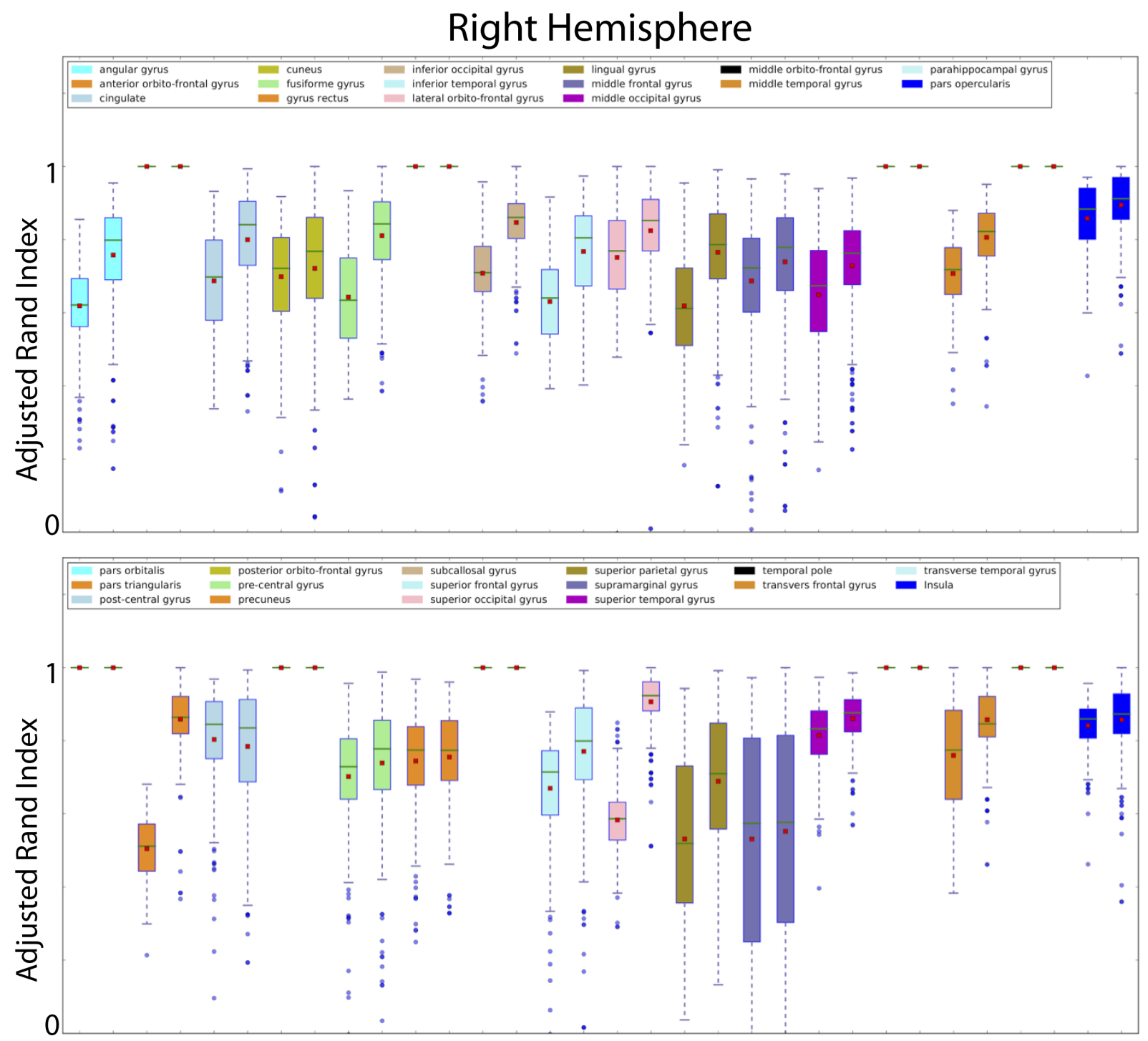

Figure 7: Adjusted rand indices (ARIs) for 60 subjects for left and right hemispheres. Each ROI has two box plots. The first (left) box plot compares atlas based sub-parcellation to rfMRI subparcellation. The second (right) box plot compares rfMRI based sub-parcellations across sessions.

\section{DISCUSSION}

The aim of this work was to develop a new brain atlas that has two levels of parcellation. The anatomical BCI-DNI atlas provides a parcellation of cortex based on established anatomical sulcal and gyral patterns. The hybrid USCBrain atlas then provides a refined parcellation that attaches subgyral labels based on functional connectivity using resting fMRI data from 40 subjects of the Human Connectome Project (HCP). 
Subdelineations were determined according to measurements of rfMRI connectivity. Parcellations of individual subjects yielded reasonable results in our validation studies based on 60 additional HCP subjects (Figure 7), which established the level of consistency of these functional boundaries for each region. In order to quantify expected intersubject variability, we also provided a probabilistic agreement map (Figure 6).

\subsection{Brain Asymmetry}

A degree of asymmetry was observed between the right and left hemisphere in both the curvature patterns that determined the boundaries in the BCI-DNI atlas (Figure 1) as well as the functionally driven boundaries in the USCBrain atlas (Figure 4). Notably, the functional boundaries subdividing the angular gyrus and its surrounding connected gyrus, as well as the middle and inferior temporal gyrus, were more asymmetric based on the topographical location of the boundaries. Regions of the medial and dorsolateral prefrontal cortex, including the cingulate gyrus and precentral gyrus, were less asymmetric. However, all pairs of homologous regions in the brain showed some degree of asymmetry (homologous boundaries jittered anywhere from $1 \mathrm{~mm}$ to $>1 \mathrm{~cm}$ ), as should be expected in human brain anatomy. Symmetry in the number of clusters each gyrus was subdivided into was enforced in the USCBrain atlas. In all four gyri where the silhoutte analysis gave differing number of subdivisions for left and right hemispheres, reasonable results, in terms of distinct connectivity patterns, good intersubject agreement, and sihoutte values relative to their maximum, were observed after matching the number of subdivisions as described in Section 2.2.2.

\subsection{Region Size}

Silhouette scores (Section 2.2.2) were the main factor that we used to determine the optimal number of subdivisions for each cortical gyrus. Most large ROIs, such as the cingulate gyrus and the middle frontal gyrus, were subdivided into 2 or 3 regions, while most small ROIs, such as the transverse frontal gyrus and pars opercularis, were subdivided into 2 regions or not divided at all (see Tables $3 \& 4$ in Appendix). Silhouette scores consistently dropped after 2 or 3 subdivisions. The USCBrain atlas resulted in 130 unique cortical ROIs and 65 cortical ROIs per hemisphere. While it has been proposed in earlier literature that the cortex consists of an estimated 180 unique functional regions per hemisphere (Nieuwenhuys, 2013), the silhouette analysis used here indicates support for only the 130 cortical regions based on the rfMRI data. Validating these regions is a current challenge in this field as we do not have cytoarchitectonic information at a fine enough resolution for the whole brain that is representative of the population to validate finer parcellations. Additionally, we considered the practical uses of this atlas along with the current challenges of signal acquisition of fMRI data, which has limited accuracy and sensitivity. The chances of misidentification of functional boundaries become increasingly error-prone as ROIs become smaller.

\subsection{Intersubject Variability}

The results comparing consistency in Figure 7 indicate, perhaps unsurprisingly, that subparcellation based on repeated within-subject rfMRI studies yields more consistent individual results than that obtained by mapping the USCBrain atlas with gyral subdivisions to the individual 
subject reflecting individualized functional parcellations. However, as noted above, rfMRI data are frequently unavailable. The observation that the ARIs for the majority of ROIs fall within average interquartile range when compared to repeated functional parcellations indicates that they represent meaningful subdivisions of these gyri. This is further supported by the agreement maps shown in Figure 6, where it is clear that for the great majority of ROIs, individual differences in functional parcellations occur close to the boundaries of the subdivisions. This variability tended to be limited to a few millimeters in most cases and reflected the typical variability we see in brain anatomy across the human population. Furthermore, a single atlas, regardless of the data used, cannot capture individual variability in functional boundaries. For that purpose an individualized parcelation is required as described, for example, in (Chong et al., 2017; Wang et al., 2015). Given the absence of anatomical landmarks to guide gyral subdivisions, use of the rfMRI based subdivision here serves as a workable surrogate. The plots in Figure 7, and probabilistic maps included with the atlas, provide the user with an indication of the degree to which these subdivisions reflect preservation of distinct functional areas within gyri.

The bilateral insula, cingulate gyrus, and post-central gyrus had very little intersubject variability. The insula's anterior and posterior regions are divided by its principal sulcus, which can be identified very easily (Bauernfeind et al., 2013; Damasio, 2005) and have very distinct functional properties. Similarly, the cingulate gyrus's 3 subdivisions have been well described in the literature and have shown to have very functionally distinct properties (Vogt et al., 1987). A few regions showed significantly lower performance (high variability/less consistency) using anatomical parcellation in comparison to functionally-based parcellation, indicating high intersubject variability in the corresponding functional boundaries. These regions, such as the right pars triangularis, right superior occipital gyrus, left lingual gyrus, and right superior parietal gyrus, are expected to have less reliable identification in individual subjects

Conversely, bilateral superior parietal gyrus, right supramarginal gyrus, left middle frontal gyrus and left superior occipital gyrus had large confidence intervals and low medians for the ARIs showing lower repeatability in Figure 7. Regions such as the pars triangularis, angular gyrus, and temporal lobe also tended to have less agreement across the 40 subjects in their functional boundaries in Figure 6. This lack of agreement can be explained by normal variance observed across subjects in these regions. The pars triangularis is determined by 2 sulcal branches which extend from the Sylvian sulcus which can be hard to identify and, in some cases, only a single branch may be present in an individual (Damasio, 2005; Keller et al., 2007; Ono et al., 1990). The superior parietal gyrus extends into the angular gyrus, which connects into the occipital lobe. This region is a multisensory cortex, which has very complex connection patterns and functional properties. Finally, the temporal lobe's sulcal patterns have been noted to have some of the highest complexities and variability across subjects (Ono et al., 1990). We expected to see less consistency in frontal lobe due to the presence of susceptibility-related field distortion and in fMRI data. However, this does not seem to be the case, reflecting the high quality of fMRI data and preprocessing in the HCP dataset.

Users should be more cautious when interpreting functional data in regions with high variability across the population and with less stable signal across sessions, as they may lead to studies with lower reproducibility. Therefore, we provide the confidence map in Figure 6 and ARI values in Figure 7 to give users quantitative measures of uncertainty for the USCBrain atlas. The agreement 
probability map shown in Figure 6 can be transferred to the subject using the one-to-one correspondence established by registration. This probability map in conjunction with the labels gives probabilistic labeling of subjects where the transferred agreement map indicates the reliability of the subdivisions. This agreement probability map is packaged with the USCBrain atlas.

\subsection{Atlas Usage}

The BCI-DNI atlas and the USCBrain atlas are intended to be used for coregistration and segmentation of individual $\mathrm{T} 1 \mathrm{~W}$ brain images. Brain labeling or coregistration is a necessary preprocessing step for many studies performing group comparisons, correlations, and regional brain analysis. Individual T1-weighted images can be used to register images of other contrast (e.g., fMRI or DTI) from the same individual using simpler rigid registration methods. Labels, as well as the agreement probability maps, can therefore be transferred from the atlas to any of the subject images to identify anatomical and functional regions. Alternatively, images can be transformed to the atlas space for voxel-wise analysis or vertex-wise analysis on the surface.

These atlases were specifically customized and will have optimal performance when used with the BrainSuite software package (http://brainsuite.org) (Joshi et al., 2012b, 2007; Shattuck and Leahy, 2002). BrainSuite includes a robust registration algorithm that uses anatomical information from both the surface and volume of the brain images for accurate automated coregistration which allows consistent surface and volume mapping to a labeled atlas. It uses a multi-step registration and refinement process based on morphological and image intensity features and known variations in human brain anatomy and is consistent with use of a detailed single-subject atlas. The BCI-DNI atlas is packaged with the BrainSuite software (http://brainsuite.org/download). Both the BCI-DNI and USCBrain atlases with anatomical and functional segmentations can be separately downloaded to be used with BrainSuite (http://brainsuite.org/atlases). Compatibility with Freesurfer and FSL 

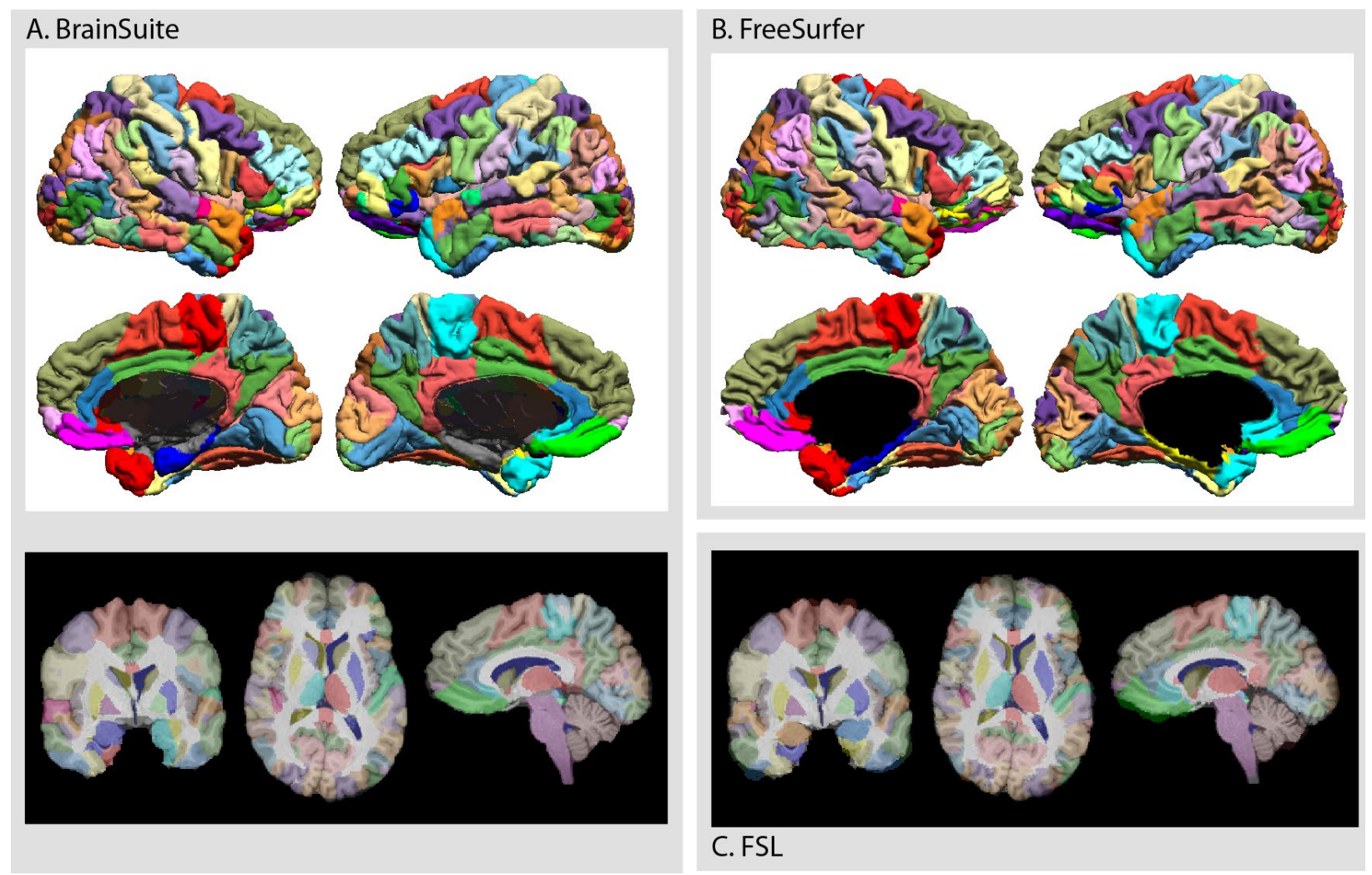

Figure 8: The atlas was used for labeling a representative subject using BrainSuite, FreeSurfer and FSL. (a) BrainSuite software can be used with the USCBrain Atlas to perform surface and volume labeling. The USCBrain Atlas can also be used with (b) FreeSurfer, to perform surface labeling, and (c) FSL (FLIRT+FNIRT) to perform volume labeling.

is provided as described in section 2.4 and compatible files and scripts are available for public use (http://brainsuite.org/using-atlases/). The BrainSuite preprocessing pipeline will output surface and volumetric labeling of individual subjects. The Freesurfer pipeline outputs surface labeling and FSL outputs volumetric labeling of T1W images. As seen in Figure 8, BrainSuite and Freesurfer yield very similar results. BrainSuite and FSL produce similar results subcortically in volumetric labeling, however, FSL tends to have more bleeding across the gyral boundaries.

\section{CONCLUSION}

In this study, we generated the anatomical BCI-DNI atlas based on single subject anatomy, which was then used as a starting point for generating the hyrid USCBrain atlas with labels based on single-subject anatomy as well as functional data from a population of 40 subjects. The parcellations defined in the atlas are based on both the known anatomical landmarks defined by gyri as well as functional subdivisions of these gyri. The intended use of this atlas is to subparcellate cortical gyri into finer sub-divisions in the absence of anatomical landmarks in applications such as neurosurgery and studies in cognitive neuroscience. The relatively high degree of intersubject labeling agreement in the validation study indicates the utility of this atlas for labeling subjects using anatomically driven coregistration. This atlas can be downloaded and used with our BrainSuite software (http://brainsuite.org/atlases). The atlas is also compatible with FreeSurfer and FSL. 


\section{ACKNOWLEDGMENT}

This work is supported by the following grants: W81XWH-18-1-061, R01 NS074980, R01 NS089212, F31NS106828, and T32 MH 111360.

The HCP data used in this work were provided by the Human Connectome Project, WU-Minn Consortium (Principal Investigators: David Van Essen and Kamil Ugurbil; 1U54MH091657) funded by the 16 NIH Institutes and Centers that support the NIH Blueprint for Neuroscience Research; and by the McDonnell Center for Systems Neuroscience at Washington University.

\section{DATA AVAILABILITY STATEMENT}

The data that support the findings of this study (USCBrain atlas) are openly available at http://brainsuite.org/uscbrainatlas.

Data Citation: Joshi et al. USCBrain atlas: http://brainsuite.org/uscbrainatlas 2019.

\section{REFERENCES}

Andersson, J.L., Jenkinson, M., Smith, S., 2007. Non-linear registration, aka spatial normalisation. FMRIB Tech. Rep. TR07JA2 22.

Bauernfeind, A.L., de Sousa, A.A., Avasthi, T., Dobson, S.D., Raghanti, M.A., Lewandowski, A.H., Zilles, K., Semendeferi, K., Allman, J.M., Craig, A.D. (Bud), Hof, P.R., Sherwood, C.C., 2013. A volumetric comparison of the insular cortex and its subregions in primates. J. Hum. Evol. 64, 263-279. https://doi.org/10.1016/j.jhevol.2012.12.003

Bhushan, C., Chong, M., Choi, S., Joshi, A.A., Haldar, J.P., 2016. Temporal Non-Local Means Filtering Reveals Real-Time Whole-Brain Cortical Interactions in Resting fMRI. PloS One 11, 1-22. https://doi.org/10.1371/journal.pone.0158504

Blumensath, T., Behrens, T.E.J., Smith, S.M., 2012. Resting-State FMRI Single Subject Cortical Parcellation Based on Region Growing, in: International Conference on Medical Image Computing and Computer-Assisted Intervention. Springer, pp. 188-195. https://doi.org/10.1007/978-3-642-33418-4_24

Calhoun, V.D., Liu, J., Adali, T., 2009. A review of group ICA for fMRI data and ICA for joint inference of imaging, genetic, and ERP data. NeuroImage 45, S163-S172. https://doi.org/10.1016/j.neuroimage.2008.10.057

Chakravarty, M.M., Bertrand, G., Hodge, C.P., Sadikot, A.F., Collins, D.L., 2006. The creation of a brain atlas for image guided neurosurgery using serial histological data. NeuroImage 30, 359-376. https://doi.org/10.1016/j.neuroimage.2005.09.041 
Chong, M., Bhushan, C., Joshi, A.A., Choi, S., Haldar, J.P., Shattuck, D.W., Spreng, R.N., Leahy, R.M., 2017. Individual parcellation of resting fMRI with a group functional connectivity prior. NeuroImage 156, 87-100. https://doi.org/10.1016/j.neuroimage.2017.04.054

Collins, D.L., Holmes, C.J., Peters, T.M., Evans, A.C., 1995. Automatic 3-D model-based neuroanatomical segmentation. Hum. Brain Mapp. 3, 190-208. https://doi.org/10.1002/hbm.460030304

Cordes, D., Haughton, V., Carew, J.D., Arfanakis, K., Maravilla, K., 2002. Hierarchical clustering to measure connectivity in fMRI resting-state data. Magn. Reson. Imaging 20, 305-317. https://doi.org/10.1016/S0730-725X(02)00503-9

Craddock, R.C., James, G.A., Iii, P.E.H., Hu, X.P., Mayberg, H.S., 2013. A whole brain fMRI atlas spatial Generated via Spatially Constrained Spectral Clustering_Craddock, James 2011 .pdf. Hum. Brain Mapp. 33, 1914-1928. https://doi.org/10.1002/hbm.21333.A

Damasio, H., 2005. Human brain anatomy in computerized images. Oxford University Press, USA.

de Amorim, R.C., Hennig, C., 2015. Recovering the number of clusters in data sets with noise features using feature rescaling factors. Inf. Sci. 324, 126-145. https://doi.org/10.1016/j.ins.2015.06.039

Desbrun, M., Polthier, K., 2007. Discrete Differential Geometry. Comput. Aided Geom. Des. 24, 427. https://doi.org/10.1016/j.cagd.2007.07.005

Desikan, R.S., Ségonne, F., Fischl, B., Quinn, B.T., Dickerson, B.C., Blacker, D., Buckner, R.L., Dale, A.M., Maguire, R.P., Hyman, B.T., others, 2006. An automated labeling system for subdividing the human cerebral cortex on MRI scans into gyral based regions of interest. Neuroimage 31, 968-980.

Dickie, D.A., Shenkin, S.D., Anblagan, D., Lee, J., Blesa Cabez, M., Rodriguez, D., Boardman, J.P., Waldman, A., Job, D.E., Wardlaw, J.M., 2017. Whole Brain Magnetic Resonance Image Atlases: A Systematic Review of Existing Atlases and Caveats for Use in Population Imaging. Front. Neuroinformatics 11. https://doi.org/10.3389/fninf.2017.00001

Do Carmo, M.P., 2016. Differential geometry of curves and surfaces: revised and updated second edition. Courier Dover Publications.

Duvernoy, H.M., 1999. The Human Brain. Springer Vienna, Vienna. https://doi.org/10.1007/9783-7091-6792-2

Essen, D.C.V., Drury, H.A., 1997. Structural and Functional Analyses of Human Cerebral Cortex Using a Surface-Based Atlas. J. Neurosci. 17, 7079-7102.

Fischl, $\quad$ B., 2012. $\quad$ FreeSurfer. $\quad$ NeuroImage $\quad 62, \quad 774-781$. https://doi.org/10.1016/j.neuroimage.2012.01.021 
Fischl, B., Sereno, M.I., Dale, A.M., 1999. Cortical Surface-Based Analysis. NeuroImage 9, 195207. https://doi.org/10.1006/nimg.1998.0396

Glasser, M.F., Coalson, T.S., Robinson, E.C., Hacker, C.D., Harwell, J., Yacoub, E., Ugurbil, K., Andersson, J., Beckmann, C.F., Jenkinson, M., Smith, S.M., Van Essen, D.C., 2016. A multi-modal parcellation of human cerebral cortex. Nature 536, 171-178. https://doi.org/10.1038/nature18933

Glasser, M.F., Goyal, M.S., Preuss, T.M., Raichle, M.E., Van Essen, D.C., 2014. Trends and properties of human cerebral cortex: correlations with cortical myelin content. NeuroImage 93 Pt 2, 165-175. https://doi.org/10.1016/j.neuroimage.2013.03.060

Glasser, M.F., Sotiropoulos, S.N., Wilson, J.A., Coalson, T.S., Fischl, B., Andersson, J.L., Xu, J., Jbabdi, S., Webster, M., Polimeni, J.R., Van Essen, D.C., Jenkinson, M., 2013. The minimal preprocessing pipelines for the Human Connectome Project. NeuroImage 80, 105-124. https://doi.org/10.1016/j.neuroimage.2013.04.127

Joshi, A.A., Chong, M., Li, J., Choi, S., Leahy, R.M., 2018. Are you thinking what I'm thinking? Synchronization of resting fMRI time-series across subjects. NeuroImage 172, 740-752. https://doi.org/10.1016/j.neuroimage.2018.01.058

Joshi, A.A., Pantazis, D., Li, Q., Damasio, H., Shattuck, D.W., Toga, A.W., Leahy, R.M., 2010. Sulcal set optimization for cortical surface registration. NeuroImage 50, 950-959. https://doi.org/10.1016/j.neuroimage.2009.12.064

Joshi, A.A., Shattuck, D.W., Damasio, H., Leahy, R.M., 2012a. Geodesic curvature flow on surfaces for automatic sulcal delineation, in: Proceedings - International Symposium on Biomedical Imaging. IEEE, pp. 430-433. https://doi.org/10.1109/ISBI.2012.6235576

Joshi, A.A., Shattuck, D.W., Leahy, R.M., 2012b. A Method for Automated Cortical Surface Registration and Labeling, in: Dawant, B.M., Christensen, G.E., Fitzpatrick, J.M., Rueckert, D. (Trans.), Biomedical Image Registration. Springer Berlin Heidelberg, Berlin, Heidelberg, pp. 180-189.

Joshi, A.A., Shattuck, D.W., Thompson, P.M., Leahy, R.M., 2007. Surface-Constrained Volumetric Brain Registration Using Harmonic Mappings. IEEE Trans Med Imaging 26, 1657-1669.

Keller, S.S., Highley, J.R., Garcia-Finana, M., Sluming, V., Rezaie, R., Roberts, N., 2007. Sulcal variability, stereological measurement and asymmetry of Broca's area on MR images. J. Anat. 211, 534-555. https://doi.org/10.1111/j.1469-7580.2007.00793.x

Kuhn, H.W., 1955. The Hungarian method for the assignment problem. Nav. Res. Logist. Q. 2, 83-97. https://doi.org/10.1002/nav.3800020109 
Li, J., Choi, S., Joshi, A.A., Wisnowski, J.L., Leahy, R.M., 2018. Global PDF-based temporal nonlocal means filtering reveals individual differences in brain connectivity, in: 2018 IEEE 15th International Symposium on Biomedical Imaging (ISBI 2018). Presented at the 2018 IEEE 15th International Symposium on Biomedical Imaging (ISBI 2018), pp. 15-19. https://doi.org/10.1109/ISBI.2018.8363513

Mailo, J., Tang-Wai, R., 2015. Insight into the precuneus: a novel seizure semiology in a child with epilepsy arising from the right posterior precuneus. Epileptic Disord. Int. Epilepsy J. Videotape 17, 321-327. https://doi.org/10.1684/epd.2015.0759

Margulies, D.S., Vincent, J.L., Kelly, C., Lohmann, G., Uddin, L.Q., Biswal, B.B., Villringer, A., Castellanos, F.X., Milham, M.P., Petrides, M., 2009. Precuneus shares intrinsic functional architecture in humans and monkeys. Proc. Natl. Acad. Sci. 106, 20069-20074. https://doi.org/10.1073/pnas.0905314106

Mazziotta, J.C., Toga, A.W., Evans, A., Fox, P., Lancaster, J., 1995. A Probabilistic Atlas of the Human Brain: Theory and Rationale for Its Development. NeuroImage 2, 89-101. https://doi.org/10.1006/nimg.1995.1012

Miller, M.B., Donovan, C.-L., Van Horn, J.D., German, E., Sokol-Hessner, P., Wolford, G.L., 2009. Unique and persistent individual patterns of brain activity across different memory retrieval tasks. NeuroImage 48, 625-635. https://doi.org/10.1016/j.neuroimage.2009.06.033

Nieuwenhuys, R., 2013. The myeloarchitectonic studies on the human cerebral cortex of the VogtVogt school, and their significance for the interpretation of functional neuroimaging data. Brain Struct. Funct. 218, 303-352. https://doi.org/10.1007/s00429-012-0460-Z

Ono, M., Kubik, S., Abernathey, C.D., 1990. Atlas of the Cerebral Sulci. Stuttgart, New York:Thieme.

Pantazis, D., Joshi, A., Jiang, J., Shattuck, D.W., Bernstein, L.E., Damasio, H., Leahy, R.M., 2010. Comparison of landmark-based and automatic methods for cortical surface registration. Neuroimage 49, 2479-2493.

Postelnicu, G., Zollei, L., Fischl, B., 2009. Combined Volumetric and Surface Registration. IEEE Trans. Med. Imaging 28, 508-522. https://doi.org/10.1109/TMI.2008.2004426

Rand, W.M., 1971. Objective Criteria for the Evaluation of Clustering Methods. J. Am. Stat. Assoc. 66, 846-850. https://doi.org/10.2307/2284239

Rousseeuw, P.J., 1987. Rousseeuw, P.J.: Silhouettes: A Graphical Aid to the Interpretation and Validation of Cluster Analysis. Comput. Appl. Math. 20, 53-65. J. Comput. Appl. Math. 20, 53-65. 
Shattuck, D.W., Leahy, R.M., 2002. BrainSuite: An Automated Cortical Surface Identification Tool. Med. Image Anal. 8, 129-142.

Shattuck, D.W., Sandor-Leahy, S.R., Schaper, K.A., andR. M. Leahy, D.A.R., Rottenberg, D.A., Leahy, R.M., 2001. Magnetic Resonance Image Tissue Classification Using a Partial volume model. NeuroImage 13, 856-876.

Shi, J., Malik, J., 2005. Normalized Cuts and Image Segmentation Normalized Cuts and Image Segmentation. IEEE Trans. Pattern Anal. Mach. Intell. 22, 888-905. https://doi.org/10.1109/CVPR.1997.609407

Talairach, J., Tournoux, P., 1988. Co-planar stereotaxic atlas of the human brain. 3-Dimensional proportional system: an approach to cerebral imaging. Thieme.

Tzourio-Mazoyer, N., Landeau, B., Papathanassiou, D., Crivello, F., Etard, O., Delcroix, N., Mazoyer, B., Joliot, M., 2002. Automated Anatomical Labeling of Activations in SPM Using a Macroscopic Anatomical Parcellation of the MNI MRI Single-Subject Brain. NeuroImage 15, 273-289. https://doi.org/10.1006/nimg.2001.0978

Van Essen, D.C., Smith, S.M., Barch, D.M., Behrens, T.E.J., Yacoub, E., Ugurbil, K., WU-Minn HCP Consortium, 2013. The WU-Minn Human Connectome Project: an overview. NeuroImage 80, 62-79. https://doi.org/10.1016/j.neuroimage.2013.05.041

Vogt, B.A., Pandya, D.N., Rosene, D.L., 1987. Cingulate cortex of the rhesus monkey: I. Cytoarchitecture and thalamic afferents. J. Comp. Neurol. 262, 256-270. https://doi.org/10.1002/cne.902620207

Wang, D., Buckner, R.L., Fox, M.D., Holt, D.J., Holmes, A.J., Stoecklein, S., Langs, G., Pan, R., Qian, T., Li, K., Baker, J.T., Stufflebeam, S.M., Wang, K., Wang, X., Hong, B., Liu, H., 2015. Parcellating cortical functional networks in individuals. Nat. Neurosci. 18, 18531860. https://doi.org/10.1038/nn.4164

Yeo, B.T.T., Krienen, F.M., Sepulcre, J., Sabuncu, M.R., Lashkari, D., Hollinshead, M., Roffman, J.L., Smoller, J.W., Zöllei, L., Polimeni, J.R., Fischl, B., Liu, H., Buckner, R.L., 2011. The organization of the human cerebral cortex estimated by intrinsic functional connectivity. J. Neurophysiol. 106, 1125-1165. https://doi.org/10.1152/jn.00338.2011

Zilles, K., Amunts, K., 2010. Centenary of Brodmann's map - conception and fate. Nat. Rev. Neurosci. 11, 139-145. https://doi.org/10.1038/nrn2776 


\section{Appendix}

\section{ROIs and sulci labeled in BCI-DNI atlas}

\begin{tabular}{|c|c|c|}
\hline \multicolumn{3}{|l|}{ Frontal Lobe } \\
\hline superior frontal gyrus & middle frontal gyrus & pars opercularis \\
\hline pars triangularis & pars orbitalis & pre-central gyrus \\
\hline transverse frontal gyrus & gyrus rectus & middle orbitofrontal gyrus \\
\hline anterior orbitofrontal gyrus & posterior orbitofrontal gyrus & lateral orbital gyrus \\
\hline paracentral lobule & cingulate gyrus & subcallosal gyrus \\
\hline \multicolumn{3}{|l|}{ Parietal Lobe } \\
\hline post-central gyrus & supramarginal gyrus & angular gyrus \\
\hline superior parietal gyrus & pre-cuneus & \\
\hline \multicolumn{3}{|l|}{ Temporal Lobe } \\
\hline temporal pole & superior temporal gyrus & transverse temporal gyrus \\
\hline middle temporal gyrus & inferior temporal gyrus & fusiform gyrus \\
\hline parahippocampal gyrus & hippocampus* & amygdala* \\
\hline \multicolumn{3}{|l|}{ Occipital Lobe } \\
\hline superior occipital gyrus & middle occipital gyrus & inferior occipital gyrus \\
\hline lingual gyrus & cuneus & \\
\hline \multicolumn{3}{|l|}{ Subcortical and Others } \\
\hline caudate nucleus* & putamen* & globus pallidus* \\
\hline nucleus accumbens* & thalamus* & inferior colliculus* \\
\hline superior colliculus* & mamillary body* & pineal gland* \\
\hline lateral ventricles* & third ventricle* & fourth ventricle* \\
\hline cerebral aqueduct* & brainstem* & corpus callosum* \\
\hline cerebellum* & insula & \\
\hline
\end{tabular}

Table 1. 95 Regions of interests (ROI) labeled on the BCI-DNI anatomical brain atlas. 66 of these regions are cortical ROIs and are labeled on the surface. *indicates non-cortical ROIs that are not labeled on the surface. 


\begin{tabular}{|l|l|}
\hline central sulcus* & precentral sulcus* \\
\hline superior frontal sulcus* & inferior frontal sulcus* \\
\hline ascending branch of the Sylvian fissure* & horizontal branch of the Sylvian fissure* \\
\hline diagonal sulcus & lateral orbital sulcus* \\
\hline frontomarginal sulcus* & cingulate sulcus* \\
\hline paracentral sulcus* & superior supra orbital sulcus* \\
\hline inferior supra orbital sulcus & olfactory or medial orbital sulcus* \\
\hline H shaped sulci, mesial & H shaped sulci, lateral \\
\hline H shaped sulci, transverse & sylvian fissure terminal split $*$ \\
\hline superior temporal sulcus* & inferior temporal sulcus* \\
\hline occipito temporal sulcus* & collateral or medial occipito temporal sulcus* \\
\hline transverse temporal sulcus* & circular sulcus* \\
\hline postcentral sulcus* & intraparietal sulcus* \\
\hline primary sulcus of Jensen & secondary sulcus of Jensen \\
\hline parieto occipital sulcus* & subparietal sulcus* \\
\hline calcarine sulcus* & calcarine sulcus terminal T \\
\hline transverse occipital sulcus* & superior lateral occipital sulcus \\
\hline inferior lateral occipital sulcus & anterior occipital sulcus \\
\hline
\end{tabular}

Table 2. 26 sulci labeled on each hemisphere of the BCI-DNI anatomical brain atlas according to the BrainSuite curve protocol (http://neuroimage.usc.edu/CurveProtocol.html). Additional sulci are marked on the second set of curves totaling 39 sulci on the left hemisphere and 37 sulci on the right hemisphere. *indicates sulci as described in the original BrainSuite curve protocol. 


\section{Silhouette Coefficients}

\begin{tabular}{|c|c|c|c|c|c|}
\hline \multirow[b]{2}{*}{ ROI (Left hemisphere) } & \multicolumn{5}{|c|}{ Average Silhouette Score } \\
\hline & $\begin{array}{l}2 \\
\text { Clusters } \\
\end{array}$ & $\begin{array}{l}3 \\
\text { Clusters } \\
\end{array}$ & $\begin{array}{l}4 \\
\text { Clusters }\end{array}$ & $\begin{array}{l}5 \\
\text { Clusters } \\
\end{array}$ & $\begin{array}{l}6 \\
\text { Clusters } \\
\end{array}$ \\
\hline Precuneus & 0.46 & 0.32 & 0.25 & 0.253 & 0.26 \\
\hline Angular gyrus & 0.54 & 0.42 & 0.36 & 0.33 & 0.30 \\
\hline Anterior orbito-frontal gyrus & 0.21 & 0.20 & 0.23 & 0.21 & 0.24 \\
\hline Cingulate & 0.38 & 0.46 & 0.36 & 0.33 & 0.28 \\
\hline Cuneus & 0.30 & 0.29 & 0.28 & 0.27 & 0.24 \\
\hline Fusiforme gyrus & 0.49 & 0.27 & 0.30 & 0.21 & 0.22 \\
\hline Gyrus rectus & 0.41 & 0.28 & 0.22 & 0.23 & 0.15 \\
\hline Inferior occipital gyrus & 0.42 & 0.47 & 0.42 & 0.42 & 0.40 \\
\hline Inferior temporal gyrus & 0.51 & 0.38 & 0.31 & 0.30 & 0.24 \\
\hline Lateral orbito-frontal gyrus & 0.48 & 0.31 & 0.27 & 0.22 & 0.26 \\
\hline Lingual gyrus & 0.43 & 0.38 & 0.27 & 0.28 & 0.25 \\
\hline Middle frontal gyrus & 0.39 & 0.33 & 0.29 & 0.24 & 0.24 \\
\hline Middle occipital gyrus & 0.55 & 0.51 & 0.45 & 0.31 & 0.26 \\
\hline Middle orbito-frontal gyrus & 0.15 & 0.14 & 0.13 & 0.15 & 0.17 \\
\hline Middle temporal gyrus & 0.44 & 0.31 & 0.40 & 0.32 & 0.28 \\
\hline Parahippocampal gyrus & 0.21 & 0.17 & 0.18 & 0.15 & 0.16 \\
\hline Pars opercularis & 0.37 & 0.31 & 0.23 & 0.23 & 0.18 \\
\hline Pars orbitalis & 0.29 & 0.25 & 0.23 & 0.25 & 0.28 \\
\hline Pars triangularis & 0.29 & 0.34 & 0.33 & 0.31 & 0.29 \\
\hline Post-central gyrus & 0.41 & 0.38 & 0.29 & 0.32 & 0.30 \\
\hline Posterior orbito-frontal gyrus & 0.15 & 0.18 & 0.21 & 0.22 & 0.24 \\
\hline Pre-central gyrus & 0.39 & 0.30 & 0.33 & 0.29 & 0.27 \\
\hline Subcallosal gyrus & 0.13 & 0.16 & 0.24 & 0.25 & 0.25 \\
\hline Superior frontal gyrus & 0.45 & 0.26 & 0.21 & 0.23 & 0.27 \\
\hline Superior occipital gyrus & 0.41 & 0.33 & 0.31 & 0.27 & 0.22 \\
\hline Superior parietal gyrus & 0.33 & 0.33 & 0.29 & 0.33 & 0.33 \\
\hline Supramarginal gyrus & 0.48 & 0.39 & 0.35 & 0.32 & 0.31 \\
\hline Superior temporal gyrus & 0.31 & 0.28 & 0.27 & 0.31 & 0.28 \\
\hline Temporal pole & 0.22 & 0.21 & 0.20 & 0.19 & 0.17 \\
\hline Transvers frontal gyrus & 0.38 & 0.25 & 0.24 & 0.21 & 0.22 \\
\hline Transverse temporal gyrus & 0.23 & 0.17 & 0.15 & 0.19 & 0.19 \\
\hline Insula & 0.37 & 0.28 & 0.21 & 0.18 & 0.20 \\
\hline
\end{tabular}

Table 3: Silhouette coefficients for different numbers of clusters for each anatomical ROI in the left hemisphere. Final subdivisions chosen for the USCBrain atlas are labeled in red. 


\begin{tabular}{|c|c|c|c|c|c|}
\hline \multirow[b]{2}{*}{ ROI (Right hemisphere) } & \multicolumn{5}{|c|}{ Average Silhouette Score } \\
\hline & $\begin{array}{l}2 \\
\text { Clusters }\end{array}$ & \begin{tabular}{l|}
3 \\
Clusters \\
\end{tabular} & $\begin{array}{l}4 \\
\text { Clusters }\end{array}$ & $\begin{array}{l}5 \\
\text { Clusters }\end{array}$ & $\begin{array}{l}6 \\
\text { Clusters }\end{array}$ \\
\hline Precuneus & 0.48 & 0.35 & 0.22 & 0.29 & 0.25 \\
\hline Angular gyrus & 0.28 & 0.37 & 0.33 & 0.31 & 0.33 \\
\hline Anterior orbito-frontal gyrus & 0.21 & 0.18 & 0.19 & 0.20 & 0.21 \\
\hline Cingulate & 0.34 & 0.43 & 0.37 & 0.31 & 0.24 \\
\hline Cuneus & 0.36 & 0.28 & 0.28 & 0.28 & 0.25 \\
\hline Fusiforme gyrus & 0.49 & 0.29 & 0.24 & 0.21 & 0.16 \\
\hline Gyrus Rectus & 0.21 & 0.14 & 0.15 & 0.15 & 0.11 \\
\hline Inferior occipital gyrus & 0.34 & 0.36 & 0.38 & 0.39 & 0.33 \\
\hline Inferior temporal gyrus & 0.58 & 0.37 & 0.31 & 0.25 & 0.22 \\
\hline Lateral orbito-frontal gyrus & 0.29 & 0.16 & 0.20 & 0.18 & 0.17 \\
\hline Lingual gyrus & 0.34 & 0.20 & 0.28 & 0.26 & 0.25 \\
\hline Middle frontal gyrus & 0.37 & 0.27 & 0.27 & 0.23 & 0.23 \\
\hline Middle occipital gyrus & 0.40 & 0.51 & 0.36 & 0.36 & 0.32 \\
\hline Middle orbito-frontal gyrus & 0.16 & 0.15 & 0.15 & 0.15 & 0.15 \\
\hline Middle temporal gyrus & 0.36 & 0.33 & 0.38 & 0.32 & 0.26 \\
\hline Parahippocampal gyrus & 0.26 & 0.15 & 0.13 & 0.17 & 0.15 \\
\hline Pars opercularis & 0.38 & 0.26 & 0.23 & 0.19 & 0.14 \\
\hline Pars orbitalis & 0.22 & 0.20 & 0.21 & 0.22 & 0.22 \\
\hline Pars triangularis & 0.26 & 0.24 & 0.17 & 0.20 & 0.21 \\
\hline Post-central gyrus & 0.43 & 0.39 & 0.37 & 0.31 & 0.27 \\
\hline Posterior orbito-frontal gyrus & 0.13 & 0.15 & 0.16 & 0.16 & 0.18 \\
\hline Pre-central gyrus & 0.36 & 0.32 & 0.37 & 0.33 & 0.29 \\
\hline Subcallosal gyrus & 0.17 & 0.19 & 0.23 & 0.27 & 0.31 \\
\hline Superior frontal gyrus & 0.46 & 0.26 & 0.23 & 0.23 & 0.28 \\
\hline Superior occipital gyrus & 0.57 & 0.45 & 0.33 & 0.23 & 0.27 \\
\hline Superior parietal gyrus & 0.37 & 0.33 & 0.32 & 0.29 & 0.28 \\
\hline Supramarginal gyrus & 0.39 & 0.34 & 0.32 & 0.33 & 0.35 \\
\hline Superior temporal gyrus & 0.35 & 0.36 & 0.27 & 0.24 & 0.26 \\
\hline Temporal pole & 0.19 & 0.19 & 0.19 & 0.18 & 0.15 \\
\hline Transvers frontal gyrus & 0.35 & 0.22 & 0.19 & 0.19 & 0.19 \\
\hline Transverse temporal gyrus & 0.20 & 0.14 & 0.15 & 0.14 & 0.17 \\
\hline Insula & 0.30 & 0.23 & 0.20 & 0.19 & 0.14 \\
\hline
\end{tabular}

Table 4: Silhouette coefficients for different numbers of clusters for each anatomical ROI in the left hemisphere. Final subdivisions chosen for the USCBrain atlas are labeled in red. 